\title{
Robust Co-occurrence Quantification for Lexical Distributional Semantics
}

\author{
Dmitrijs Milajevs Mehrnoosh Sadrzadeh Matthew Purver \\ Queen Mary University of London \\ London, UK \\ \{d.milajevs,m.sadrzadeh,m.purver\}@qmul.ac.uk
}

\begin{abstract}
Previous optimisations of parameters affecting the word-context association measure used in distributional vector space models have focused either on highdimensional vectors with hundreds of thousands of dimensions, or dense vectors with dimensionality of a few hundreds; but dimensionality of a few thousands is often applied in compositional tasks as it is still computationally feasible and does not require the dimensionality reduction step. We present a systematic study of the interaction of the parameters of the association measure and vector dimensionality, and derive parameter selection heuristics that achieve performance across word similarity and relevance datasets competitive with the results previously reported in the literature achieved by highly dimensional or dense models.
\end{abstract}

\section{Introduction}

Words that occur in similar context have similar meaning (Harris, 1954). Thus the meaning of a word can be modeled by counting its cooccurrence with neighboring words in a corpus. Distributional models of meaning represent cooccurrence information in a vector space, where the dimensions are the neighboring words and the values are co-occurrence counts. Successful models need to be able to discriminate co-occurrence information, as not all co-occurrence counts are equally useful, for instance, the co-occurrence with the article the is less informative than with the noun existence. The discrimination is usually achieved by weighting of co-occurrence counts. Another fundamental question in vector space design is the vector space dimensionality and what neighbor words should correspond to them.

Levy et al. (2015) propose optimisations for co-occurrence-based distributional models, using parameters adopted from predictive models (Mikolov et al., 2013): shifting and context distribution smoothing. Their experiments and thus their parameter recommendations use highdimensional vector spaces with word vector dimensionality of almost $200 \mathrm{~K}$, and many recent state-of-the-art results in lexical distributional semantics have been obtained using vectors with similarly high dimensionality (Baroni et al., 2014; Kiela and Clark, 2014; Lapesa and Evert, 2014).

In contrast, much work on compositional distributional semantics employs vectors with much fewer dimensions: e.g. 2K (Grefenstette and Sadrzadeh, 2011; Kartsaklis and Sadrzadeh, 2014; Milajevs et al., 2014), 3K (Dinu and Lapata, 2010; Milajevs and Purver, 2014) or 10K (Polajnar and Clark, 2014; Baroni and Zamparelli, 2010). The most common reason thereof is that these models assign tensors to functional words. For a vector space $V$ with $k$ dimensions, a tensor $V \otimes V \cdots \otimes V$ of rank $n$ has $k^{n}$ dimensions. Adjectives and intransitive verbs have tensors of rank 2, transitive verbs are of rank 3; for coordinators, the rank can go up to 7 . Taking $k=\mathbf{2 0 0 K}$ already results in a highly intractable tensor of $\mathbf{8} \times \mathbf{1 0}^{\mathbf{1 5}}$ dimensions for a transitive verb.

An alternative way of obtaining a vector space with few dimensions, usually with just 100-500, is the use of SVD as a part of Latent Semantic Analysis (Dumais, 2004) or another models such as SGNS (Mikolov et al., 2013) and GloVe (Pennington et al., 2014). However, these models take more time to instantiate in comparison to weighting of a co-occurrence matrix, bring more parameters to explore and produce vector spaces with uninterpretable dimensions (vector space dimension interpretation is used by some lexical mod- 
els, for example, McGregor et al. (2015), and the passage from formal semantics to tensor models relies on it (Coecke et al., 2010)). In this work we focus on vector spaces that directly weight a co-occurrence matrix and report results for SVD, GloVe and SGNS from the study of Levy et al. (2015) for comparison.

The mismatch of recent experiments with nondense models in vector dimensionality between lexical and compositional tasks gives rise to a number of questions:

- To what extent does model performance depend on vector dimensionality?

- Do parameters influence $200 \mathrm{~K}$ and $1 \mathrm{~K}$ dimensional models similarly? Can the findings of Levy et al. (2015) be directly applied to models with a few thousand dimensions?

- If not, can we derive suitable parameter selection heuristics which take account of dimensionality?

To answer these questions, we perform a systematic study of distributional models with a rich set of parameters on SimLex-999 (Hill et al., 2014), a lexical similairty dataset, and test selected models on MEN (Bruni et al., 2014), a lexical relatedness dataset. These datasets are currently widely used and surpass datasets stemming from information retrieval, WordSim-353 (Finkelstein et al., 2002), and computational linguistics, RG65 (Rubenstein and Goodenough, 1965), in quantity by having more entries and in quality by attention to evaluated relations (Milajevs and Griffiths, 2016).

\section{Parameters}

\subsection{PMI variants (discr)}

Most co-occurrence weighting schemes in distributional semantics are based on point-wise mutual information (PMI, see e.g. Church and Hanks (1990), Turney and Pantel (2010), Levy and Goldberg (2014)):

$$
\operatorname{PMI}(x, y)=\log \frac{P(x, y)}{P(x) P(y)}
$$

As commonly done, we replace the infinite PMI values, ${ }^{1}$ which arise when $P(x, y)=0$, with zeroes and use PMI hereafter to refer to a weighting with this fix.

\footnotetext{
${ }^{1} \mathrm{We}$ assume that the probability of a single token is always greater than zero as it appears in the corpus at least once.
}

\begin{tabular}{ll}
\hline Parameter & Values \\
\hline \multirow{2}{*}{ Dimensionality $D$} & 1K, 2K, 3K, 5K \\
discr & 10K, 20K, 30K, 40K, 50K \\
freq & PMI, CPMI, SPMI, SCPMI \\
neg & $1, n, \log n$ \\
cds & $0.2,0.5,0.7,1,1.4,2,5,7$ \\
Similarity & global, 1, 0.75 \\
\hline
\end{tabular}

Table 1: Model parameters and their values.

An alternative solution is to increment the probability ratio by 1 ; we refer to this as compressed PMI (CPMI, see e.g. McGregor et al. (2015)):

$$
\operatorname{CPMI}(x, y)=\log \left(1+\frac{P(x, y)}{P(x) P(y)}\right)
$$

By incrementing the probability ratio by one, the PMI values from the segment of $(-\infty ; 0]$, when the joint probability $P(x, y)$ is less than the chance $P(x) P(y)$, are compressed into the segment of $(0 ; 1]$. As the result, the space does not contain negative values, but has the same sparsity as the space with PMI values.

\subsection{Shifted PMI (neg)}

Many approaches use only positive PMI values, as negative PMI values may not positively contribute to model performance and sparser matrices are more computationally tractable (Turney and Pantel, 2010). This can be generalised to an additional cutoff parameter $k$ (neg) following Levy et al. (2015), giving our third PMI variant (abbreviated as SPMI): ${ }^{2}$

$$
\mathrm{SPMI}_{\mathrm{k}}=\max (0, \operatorname{PMI}(x, y)-\log k)
$$

When $k=1$ SPMI is equivalent to positive PMI. $k>1$ increases the underlying matrix sparsity by keeping only highly associated co-occurrence pairs. $k<1$ decreases the underlying matrix sparsity by including some unassociated cooccurrence pairs, which are usually excluded due to unreliability of probability estimates (Dagan et al., 1993).

We can apply the same idea to CPMI:

$$
\mathrm{SCPMI}_{\mathrm{k}}=\max (0, \operatorname{CPMI}(x, y)-\log 2 k)
$$

\footnotetext{
${ }^{2}$ SPMI is different from CPMI because $\log \frac{P(x, y)}{P(x) P(y)}-$ $\log k=\log \frac{P(x, y)}{P(x)(P(y) k} \neq \log \left(1+\frac{P(x, y)}{P(x) P(y)}\right)$.
} 


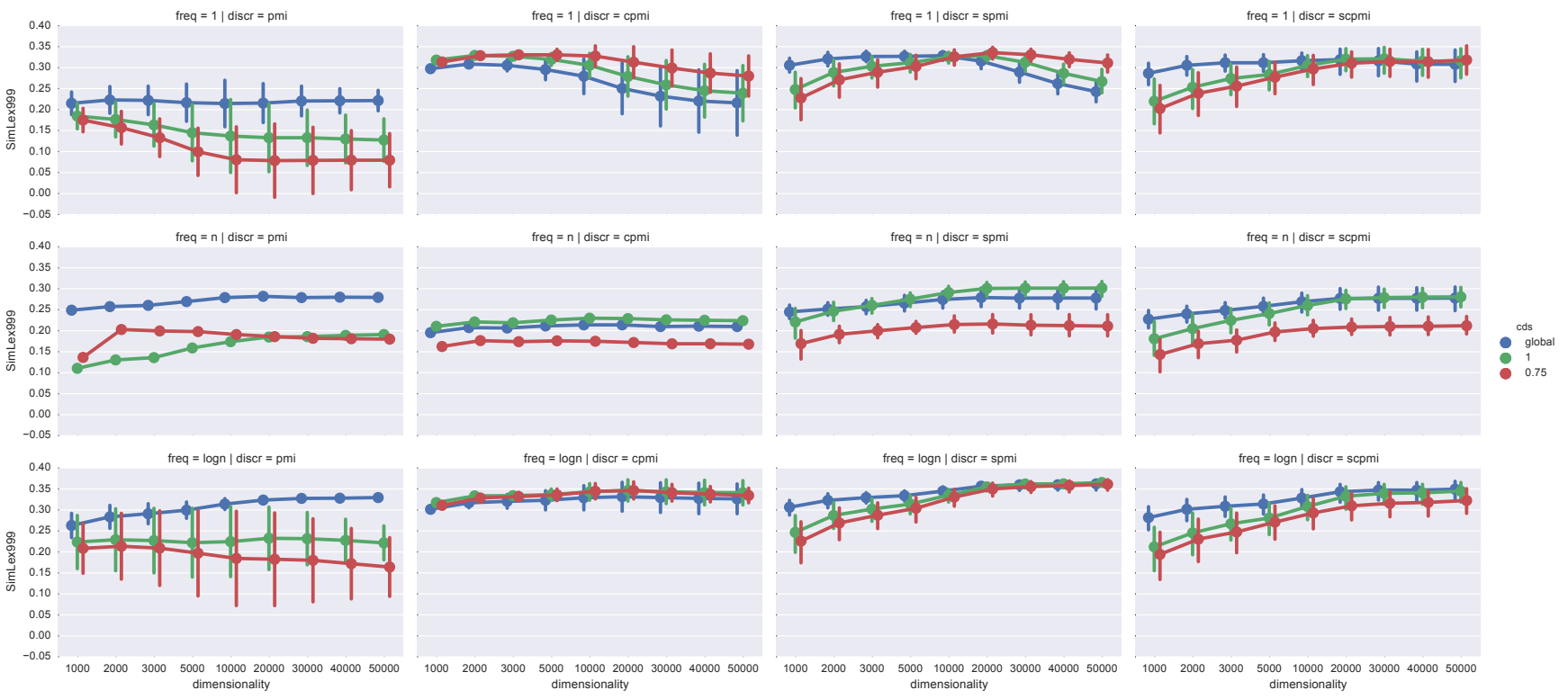

Figure 1: Effect of PMI variant (discr), smoothing (cds) and frequency weighting (freq) on SimLex-999. Error bars correspond to a 95\% confidence interval as the value is estimated by averaging over all the values of the omitted parameters: neg and similarity.

\subsection{Frequency weighting (freq)}

Another issue with PMI is its bias towards rare events (Levy et al., 2015); one way of solving this issue is to weight the value by the co-occurrence frequency (Evert, 2005):

$$
\operatorname{LMI}(x, y)=n(x, y) \operatorname{PMI}(x, y)
$$

where $n(x, y)$ is the number of times $x$ was seen together with $y$. For clarity, we refer to $n$-weighted PMIs as nPMI, nSPMI, etc. When this weighting component is set to 1, it has no effect; we can explicitly label it as 1PMI, 1SPMI, etc.

In addition to the extreme 1 and $n$ weightings, we also experiment with a $\log n$ weighting.

\subsection{Context distribution smoothing (cds)}

Levy et al. (2015) show that performance is affected by smoothing the context distribution $P(x)$ :

$$
P_{\alpha}(x)=\frac{n(x)^{\alpha}}{\sum_{c} n(c)^{\alpha}}
$$

We experiment with $\alpha=1$ (no smoothing) and $\alpha=0.75$. We call this estimation method local context probability; we can also estimate a global context probability based on the size of the corpus $C$ :

$$
P(x)=\frac{n(x)}{|C|}
$$

\subsection{Vector dimensionality $(D)$}

As context words we select the $1 \mathrm{~K}, 2 \mathrm{~K}, 3 \mathrm{~K}, 5 \mathrm{~K}$, $10 \mathrm{~K}, 20 \mathrm{~K}, 30 \mathrm{~K}, 40 \mathrm{~K}$ and $50 \mathrm{~K}$ most frequent lemmatised nouns, verbs, adjectives and adverbs. All context words are part of speech tagged, but we do not distinguish between refined word types (e.g. intransitive vs. transitive versions of verbs) and do not perform stop word filtering.

\section{Experimental setup}

Table 1 lists parameters and their values. As the source corpus we use the concatenation of Wackypedia and ukWaC (Baroni et al., 2009) with a symmetric 5-word window (Milajevs et al., 2014); our evaluation metric is the correlation with human judgements as is standard with SimLex (Hill et al., 2014). We derive our parameter selection heuristics by greedily selecting parameters (cds, neg) that lead to the highest average performance for each combination of frequency weighting, PMI variant and dimensionality $D$. Figures 1 and 2 show the interaction of cds and neg with other parameters. We also vary the similarity measure (cosine and correlation (Kiela and Clark, 2014)), but do not report results here due to space limits. ${ }^{3}$

\footnotetext{
${ }^{3}$ The results are available at http://www.eecs. qmul.ac.uk/ dm303/aclsrw2016/
} 

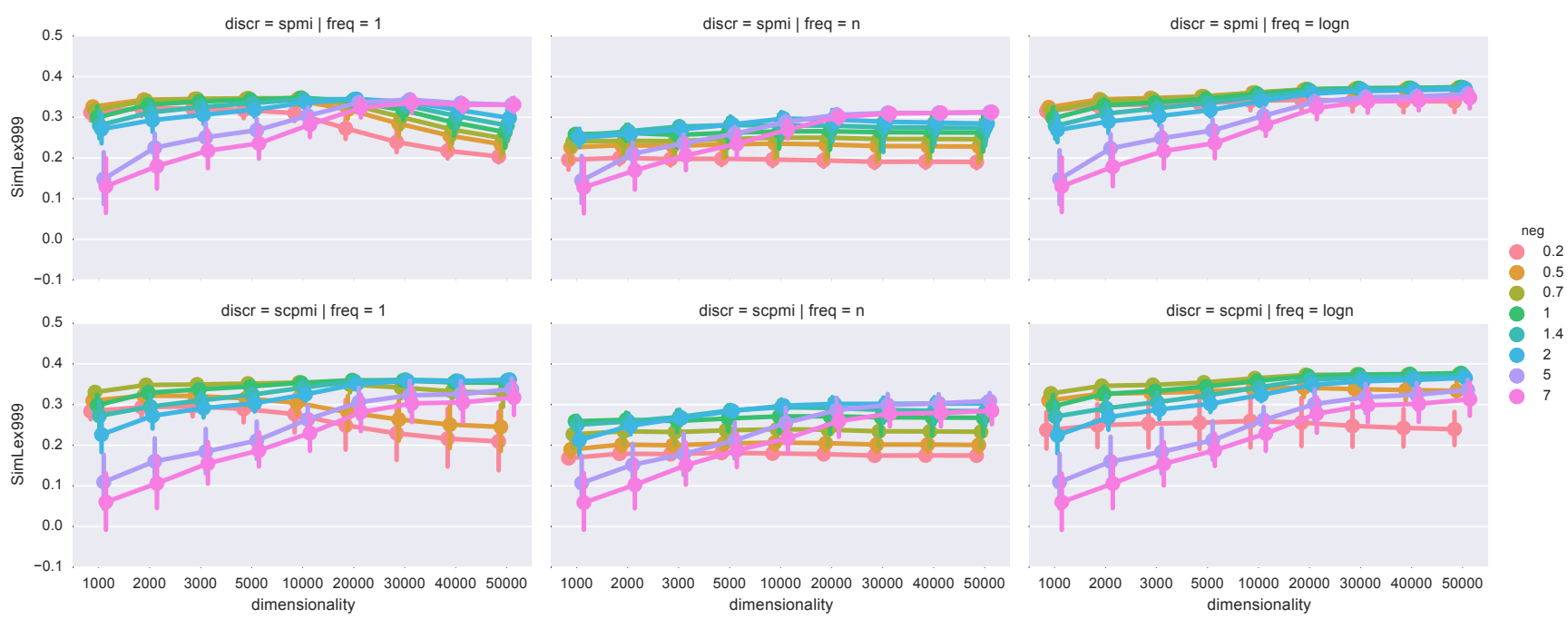

Figure 2: The behaviour of shifted PMI (SPMI) on SimLex-999. discr=spmi, freq=1 and neg= 1 corresponds to positive PMI. Error bars correspond to a 95\% confidence interval as the value is estimated by averaging over all the values of the omitted parameters: cds and similarity.

\section{Heuristics}

PMI and CPMI PMI should be used with global context probabilities. CPMI generally outperforms PMI, with less sensitivity to parameters; nCPMI and lognCPMI should be used with local context probabilities and 1CPMI should apply context distribution smoothing with $\alpha=0.75$.

SPMI 10K dimensional 1SPMI is the least sensitive to parameter selection. For models with $D>20 \mathrm{~K}$, context distribution smoothing should be used with $\alpha=0.75$; for $D<20 \mathrm{~K}$, it is beneficial to use global context probabilities. Shifting also depends on the dimensionality: models with $D<20 \mathrm{~K}$ should set $k=0.7$, but higherdimensional models should set $k=5$. There might be a finer-grained $k$ selection criteria; however, we do not report this to avoid overfitting.

lognSPMI should be used with global context probabilities for models with $D<20 \mathrm{~K}$. For higher-dimensional spaces, smoothing should be applied with $\alpha=0.75$, as with 1 SPMI. Shifting should be applied with $k=0.5$ for models with $D<20 \mathrm{~K}$, and $k=1.4$ for $D>20 \mathrm{~K}$. In contrast to 1SPMI, which might require change of $k$ as the dimensionality increases, $k=1.4$ is a much more robust choice for lognSPMI.

nSPMI gives good results with local context probabilities $(\alpha=1)$. Models with $D<20 \mathrm{~K}$ should use $k=1.4$, otherwise $k=5$ is preferred.
SCPMI With 1SCPMI and $D<20 \mathrm{~K}$, global context probability should be used, with shifting set to $k=0.7$. Otherwise, local context probability should be used with $\alpha=0.75$ and $k=2$.

With nSCPMI and $D<20 \mathrm{~K}$, global context probability should be used with $k=1.4$. Otherwise, local context probability without smoothing and $k=5$ is suggested.

For lognSCPMI, models with $D<20 \mathrm{~K}$ should use global context probabilities and $k=$ 0.7; otherwise, local context probabilities without smoothing should be preferred with $k=1.4$.

\section{Evaluation of heuristics}

We evaluate these heuristics by comparing the performance they give on SimLex-999 against that obtained using the best possible parameter selections (determined via an exhaustive search at each dimensionality setting). We also compare them to the best scores reported by Levy et al. (2015) for their model (PMI and SVD), word2vec-SGNS (Mikolov et al., 2013) and GloVe (Pennington et al., 2014)—see Figure 3a, where only the betterperforming SPMI and SCPMI are shown.

For lognPMI and lognCPMI, our heuristics pick the best possible models. For lognSPMI, where performance variance is low, the heuristics do well, giving a performance of no more than 0.01 points below the best configuration. For 1SPMI and nSPMI the difference is higher. With $\operatorname{lognSCPMI}$ and 1SCPMI, the heuristics follow 

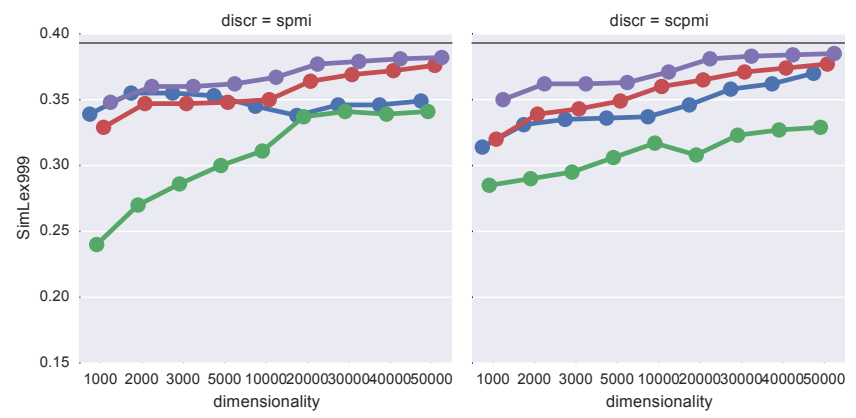

(a) SimLex-999. PPMI: 0.393, SVD: 0.432, SGNS: 0.438, GloVe: 0.398 . This work: 0.385 .
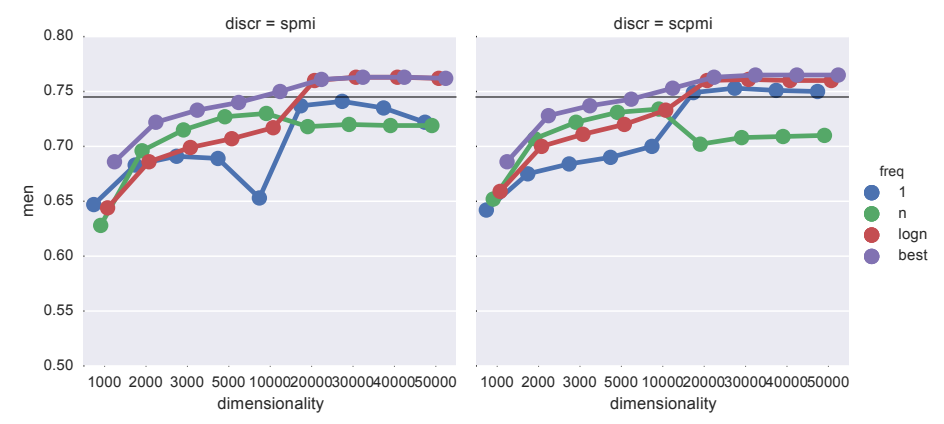

(c) MEN. PPMI: 0.745, SVD: 0.778, SGNS: 0.774, GloVe: 0.729. This work: 0.765 .

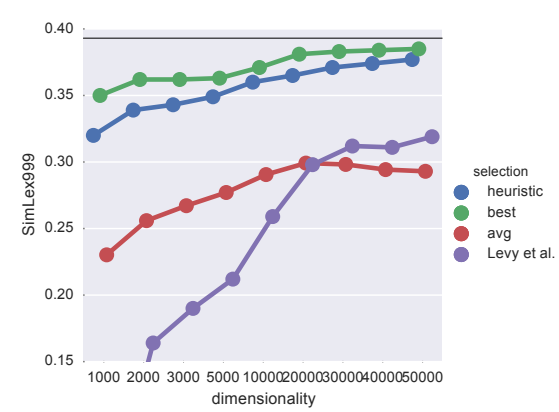

(b)

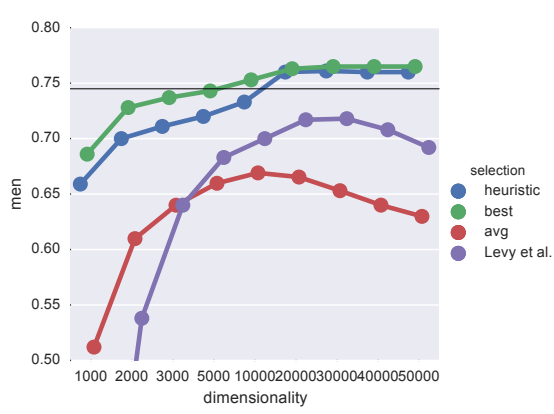

(d)

Figure 3: Best configurations. The black lines show the best count models (PPMI) reported by Levy et al. (2015). We also give our best score, SVD, SGNS and GloVe numbers from that study for comparison. On the right, our heuristic in comparison to the best and average results together with the models selected using the recommendations presented in Levy et al. (2015).

the best selection, but with a wider gap than the SPMI models. In general $n$-weighted models do not perform as well as others.

Overall, $\log n$ weighting should be used with PMI, CPMI and SCPMI. High-dimensional SPMI models show the same behaviour, but if $D<$ $10 \mathrm{~K}$, no weighting should be applied. SPMI and SCPMI should be preferred over CPMI and PMI. As Figure $3 b$ shows, our heuristics give performance close to the optimum for any dimensionality, with a large improvement over both an average parameter setting and the parameters suggested by Levy et al. (2015) in a high-dimensional setting. ${ }^{4}$

Finally, to see whether the heuristics transfer robustly, we repeat this comparison on the MEN dataset (see Figures 3c, 3d). Again, PMI and CPMI follow the best possible setup, with SPMI and SCPMI showing only a slight drop below ideal performance; and again, the heuristic settings give performance close to the optimum, and significantly higher than average or standard parameters.

\footnotetext{
${ }^{4}$ Our results using Levy et al. (2015)'s parameters differ slightly from theirs due to different window sizes (5 vs 2 ).
}

\section{Conclusion}

This paper presents a systematic study of cooccurrence quantification focusing on the selection of parameters presented in Levy et al. (2015). We replicate their recommendation for high-dimensional vector spaces, and show that with appropriate parameter selection it is possible to achieve comparable performance with spaces of dimensionality of $1 \mathrm{~K}$ to $50 \mathrm{~K}$, and propose a set of model selection heuristics that maximizes performance. We foresee the results of the paper are generalisable to other experiments, since model selection was performed on a similarity dataset, and was additionally tested on a relatedness dataset.

In general, model performance depends on vector dimensionality (the best setup with 50K dimensions is better than the best setup with $1 \mathrm{~K}$ dimensions by 0.03 on SimLex-999). Spaces with a few thousand dimensions benefit from being dense and unsmoothed ( $k<1$, global context probability); while high-dimensional spaces are better sparse and smooth $(k>1, \alpha=0.75)$. However, for unweighted and $n$-weighted models, these heuristics do not guarantee the best possible result because 


\begin{tabular}{lll}
\hline Model & SimLex-999 & MEN \\
\hline PPMI $^{*}$ & 0.393 & 0.745 \\
SVD $^{*}$ & 0.432 & 0.778 \\
SGNS* & 0.438 & 0.774 \\
GloVe* & 0.398 & 0.729 \\
This work & 0.385 & 0.765 \\
\hline
\end{tabular}

Table 2: Our model in comparison to the previous work. On the similarity dataset our model is 0.008 points behind a PPMI model, however on the relatedness dataset 0.020 points above. Note the difference in dimensionality, source corpora and window size. SVD, SGNS and GloVe numbers are given for comparison. ${ }^{*}$ Results reported by Levy et al. (2015).

of the high variance of the corresponding scores. Based on this we suggest to use lognSPMI or $\operatorname{lognSCPMI}$ with dimensionality of at least $20 \mathrm{~K}$ to ensure good performance on lexical tasks.

There are several directions for the future work. Our experiments show that models with a few thousand dimensions are competitive with more dimensional models, see Figure 3. Moreover, for these models, unsmoothed probabilities give the best result. It might be the case that due to the large size of the corpus used, the probability estimates for the most frequent words are reliable without smoothing. More experiments need to be done to see whether this holds for smaller corpora.

The similarity datasets are transferred to other languages (Leviant and Reichart, 2015). The future work might investigate whether our results hold for languages other than English.

The qualitative influence of the parameters should be studied in depth with extensive error analysis on how parameter selection changes similarity judgements.

\section{Acknowledgements}

We thank Ann Copestake for her valuable comments as part of the ACL SRW mentorship program and the anonymous reviewers for their comments. Support from EPSRC grant EP/J002607/1 is gratefully acknowledged by Dmitrijs Milajevs and Mehrnoosh Sadrzadeh. Matthew Purver is partly supported by ConCreTe: the project ConCreTe acknowledges the financial support of the Future and Emerging Technologies (FET) programme within the Seventh Framework Pro- gramme for Research of the European Commission, under FET grant number 611733.

\section{References}

Marco Baroni and Roberto Zamparelli. 2010. Nouns are vectors, adjectives are matrices: Representing adjective-noun constructions in semantic space. In Proceedings of the 2010 Conference on Empirical Methods in Natural Language Processing, EMNLP '10, pages 1183-1193, Stroudsburg, PA, USA. Association for Computational Linguistics.

Marco Baroni, Silvia Bernardini, Adriano Ferraresi, and Eros Zanchetta. 2009. The wacky wide web: a collection of very large linguistically processed web-crawled corpora. Language Resources and Evaluation, 43(3):209-226.

Marco Baroni, Georgiana Dinu, and Germán Kruszewski. 2014. Don't count, predict! a systematic comparison of context-counting vs. context-predicting semantic vectors. In Proceedings of the 52nd Annual Meeting of the Association for Computational Linguistics (Volume 1: Long Papers), pages 238-247, Baltimore, Maryland, June. Association for Computational Linguistics.

Elia Bruni, Nam Khanh Tran, and Marco Baroni. 2014. Multimodal distributional semantics. J. Artif. Int. Res., 49(1):1-47, January.

Kenneth Ward Church and Patrick Hanks. 1990. Word association norms mutual information, and lexicography. Computational Linguistics, 16(1):22-29.

Bob Coecke, Mehrnoosh Sadrzadeh, and Stephen Clark. 2010. Mathematical foundations for a compositional distributional model of meaning. CoRR, abs/1003.4394.

Ido Dagan, Shaul Marcus, and Shaul Markovitch. 1993. Contextual word similarity and estimation from sparse data. In Proceedings of the 31st Annual Meeting on Association for Computational Linguistics, ACL '93, pages 164-171, Stroudsburg, PA, USA. Association for Computational Linguistics.

Georgiana Dinu and Mirella Lapata. 2010. Measuring distributional similarity in context. In Proceedings of the 2010 Conference on Empirical Methods in Natural Language Processing, EMNLP' 10, pages 1162-1172, Stroudsburg, PA, USA. Association for Computational Linguistics.

Susan T. Dumais. 2004. Latent semantic analysis. Annual Review of Information Science and Technology, 38(1):188-230.

Stefan Evert. 2005. The statistics of word cooccurrences: word pairs and collocations. Ph.D. thesis, Universitt Stuttgart, Holzgartenstr. 16, 70174 Stuttgart. 
Lev Finkelstein, Evgeniy Gabrilovich, Yossi Matias, Ehud Rivlin, Zach Solan, Gadi Wolfman, and Eytan Ruppin. 2002. Placing search in context: The concept revisited. ACM Trans. Inf. Syst., 20(1):116131, January.

Edward Grefenstette and Mehrnoosh Sadrzadeh. 2011. Experimental support for a categorical compositional distributional model of meaning. In Proceedings of the Conference on Empirical Methods in Natural Language Processing, EMNLP' 11, pages 1394-1404, Stroudsburg, PA, USA. Association for Computational Linguistics.

Z.S. Harris. 1954. Distributional structure. Word.

Felix Hill, Roi Reichart, and Anna Korhonen. 2014. Simlex-999: Evaluating semantic models with (genuine) similarity estimation. arXiv preprint arXiv:1408.3456.

Dimitri Kartsaklis and Mehrnoosh Sadrzadeh. 2014. A study of entanglement in a categorical framework of natural language. In Proceedings of the 11th Workshop on Quantum Physics and Logic (QPL), Kyoto, Japan, June.

Douwe Kiela and Stephen Clark. 2014. A systematic study of semantic vector space model parameters. In Proceedings of the 2nd Workshop on Continuous Vector Space Models and their Compositionality (CVSC), pages 21-30, Gothenburg, Sweden, April. Association for Computational Linguistics.

Gabriella Lapesa and Stefan Evert. 2014. A large scale evaluation of distributional semantic models: $\mathrm{Pa}-$ rameters, interactions and model selection. Transactions of the Association for Computational Linguistics, 2:531-545.

Ira Leviant and Roi Reichart. 2015. Judgment language matters: Multilingual vector space models for judgment language aware lexical semantics. CoRR, abs/1508.00106.

Omer Levy and Yoav Goldberg. 2014. Neural word embedding as implicit matrix factorization. In Z. Ghahramani, M. Welling, C. Cortes, N.D. Lawrence, and K.Q. Weinberger, editors, Advances in Neural Information Processing Systems 27, pages 2177-2185. Curran Associates, Inc.

Omer Levy, Yoav Goldberg, and Ido Dagan. 2015. Improving distributional similarity with lessons learned from word embeddings. Transactions of the Association for Computational Linguistics, 3:211-225.
Stephen McGregor, Kat Agres, Matthew Purver, and Geraint Wiggins. 2015. From distributional semantics to conceptual spaces: A novel computational method for concept creation. Journal of Artificial General Intelligence, 6(1):55-86, December.

Tomas Mikolov, Kai Chen, Greg Corrado, and Jeffrey Dean. 2013. Efficient estimation of word representations in vector space. arXiv preprint arXiv:1301.3781.

Dmitrijs Milajevs and Sascha Griffiths. 2016. Treating similarity with respect: How to evaluate models of meaning? CoRR, abs/1605.04553.

Dmitrijs Milajevs and Matthew Purver. 2014. Investigating the contribution of distributional semantic information for dialogue act classification. In Proceedings of the 2nd Workshop on Continuous Vector Space Models and their Compositionality (CVSC), pages 40-47, Gothenburg, Sweden, April. Association for Computational Linguistics.

Dmitrijs Milajevs, Dimitri Kartsaklis, Mehrnoosh Sadrzadeh, and Matthew Purver. 2014. Evaluating neural word representations in tensor-based compositional settings. In Proceedings of the 2014 Conference on Empirical Methods in Natural Language Processing (EMNLP), pages 708-719, Doha, Qatar, October. Association for Computational Linguistics.

Jeffrey Pennington, Richard Socher, and Christopher Manning. 2014. Glove: Global vectors for word representation. In Proceedings of the 2014 Conference on Empirical Methods in Natural Language Processing (EMNLP), pages 1532-1543, Doha, Qatar, October. Association for Computational Linguistics.

Tamara Polajnar and Stephen Clark. 2014. Improving distributional semantic vectors through context selection and normalisation. In Proceedings of the 14th Conference of the European Chapter of the Association for Computational Linguistics, pages 230238, Gothenburg, Sweden, April. Association for Computational Linguistics.

Herbert Rubenstein and John B. Goodenough. 1965. Contextual correlates of synonymy. Commun. ACM, 8(10):627-633, October.

Peter D. Turney and Patrick Pantel. 2010. From frequency to meaning: Vector space models of semantics. J. Artif. Int. Res., 37(1):141-188, January. 